\title{
Stressful Event Exposure and PTSD Symptoms are Related to Differences in Safety Memory
}

\author{
Samantha Goldberg ${ }^{1 *}$, Rachel John², Elizabeth Duval ${ }^{3}$
}

Many individuals experience traumatic and stressful events, but very few develop post-traumatic stress disorder (PTSD). Research focused on people with PTSD and trauma-exposed healthy controls has examined how learning and memory processes may be associated with PTSD symptoms. While there is a growing literature focused on factors associated with PTSD development, fewer studies investigate relationships between stressful event exposure and learning and memory processes in healthy individuals. We aimed to study relationships between stressful event exposure, PTSD symptoms, and neural activity underlying performance during a fear extinction recall task. Participants $(N=22)$ included people with PTSD and healthy adults with and without trauma exposure. Participants were conditioned to threat in one context (e.g., living room), followed by extinction (safety learning) in another context (e.g., office). The next day, participants completed extinction recall (extinguished conditioned stimulus was presented again in the safe context) during fMRI scanning. Across all participants, there were negative relationships between extinction recall performance and stressful event exposure and post-traumatic stress symptom (PTSS) severity. In individuals with PTSD, there was a negative relationship between symptom severity and hippocampal activity during extinction recall. Our results highlight trauma- and PTSD-related differences in hippocampal function associated with learning and memory. These findings suggest that trauma exposure is associated with differences in cognition even in healthy adults and support the examination of trauma and PTSS on a continuum, in people with and without PTSD.

\section{INTRODUCTION}

Posttraumatic stress disorder (PTSD) is a trauma- and stressor-related disorder hallmarked by intrusive and often debilitating symptoms following exposure to a traumatic event. PTSD is characterized by pervasive fear and hypervigilance, which continue even in contexts where these reactions are not appropriate (American Psychiatric Association, 2013). To be diagnosed with PTSD, the Diagnostic and Statistical Manual of Mental Disorders (DSM-5) requires that the individual experienced a "criterion A" traumatic event, defined as direct or indirect exposure to "actual or threatened death, serious injury, or sexual violence," (American Psychiatric Association, 2013). In the U.S., an estimated 80

Address correspondance to:

'University of Michigan Department of Psychiatry 4250 Plymouth Rd, Ann Arbor, MI 48109

${ }^{2}$ San Diego State University Department of

Psychology 5500 Campanile Drive San Diego, CA

92182

${ }^{3}$ University of Michigan Department of Psychiatry 4250

Plymouth Rd, Ann Arbor, MI 48109

*sgberg@med.umich.edu

Submission date: November 2019

Acceptance date: June 2020

Publication date: January 2021 percent of the population will experience one or more criterion - A traumatic events in their lifetime, and less than 10 percent of those exposed to a traumatic event will develop PTSD. This is consistent among other countries, including those that have lower estimations of exposure to traumatic events (Breslau, 2009). This disparity between exposure to a traumatic event and development of PTSD brings about questions regarding what factors may be associated with risk for developing the disorder in those exposed to a traumatic event.

There is a large amount of research focused on structural and functional differences in brain areas primarily involved in memory, particularly the hippocampus, in people with PTSD compared to trauma-exposed controls, and/or nontrauma exposed controls (Gurvits et al., 1996; Gilbertson et al., 2002; Carrión et al., 2009; Milad et al., 2009; Woon et al., 2010; Logue et al., 2017). Smaller hippocampal volume has been associated with a PTSD diagnosis when compared to trauma-exposed control groups, and smaller hippocampal volume has been correlated with greater chronic PTSD symptom severity (Gurvits et al., 1996; Gilbertson et al., 2002; Apfel et al., 2011; Logue et al., 2017; Xie et al., 2017).

In addition to structural differences, functional neuroimaging studies report that people with PTSD have reduced performance on declarative memory tasks (Dickie et al., 2008; Carrión et al., 2009), and show less hippocampal activity during retrieval in some of these tasks compared to healthy controls (Carrión et al., 2009). Evidence suggests 
that hippocampal function during learning and memory tasks is associated with PTSD symptoms. For instance, Carrión et al. (2009) found a correlation between reduced hippocampal activation and greater avoidance symptoms in PTSD. Since the hippocampus is important in memory processes (Acheson et al., 2011), the findings from these studies point to deficits in hippocampal function in people with PTSD.

There are also findings suggesting smaller hippocampal volume in trauma-exposed groups without PTSD, compared to non-trauma-exposed groups (Woon et al., 2010). Longitudinal imaging work in soldiers has found stress-induced reductions in hippocampal volume, even in those who had not developed PTSD (Admon et al., 2012). In addition to hippocampal differences related to trauma exposure, there is evidence that general stress (not specifically trauma) exposure is associated with smaller hippocampal volume (Chiang et al., 2015). Since brain volume is related to function (Qing and Gong, 2016), the findings of smaller hippocampal volumes in those who have been exposed to trauma and stress, both with and without PTSD, suggest there may also be differences in hippocampal function in healthy people who have been exposed to trauma and stress, compared to those who have not.

Even without a PTSD diagnosis, there is evidence to support that there are differences in memory performance and neural activity in people with exposure to stressful and/or traumatic events compared to non-exposed individuals. For example, performance on hippocampal-dependent memory tasks has been found to be related to the amount of stressful event exposure in trauma-exposed controls (John and Duval, 2018). Additionally, evidence suggests that people who have been exposed to more stress throughout their lives, regardless of trauma-exposure, have deficits on hippocampaldependent memory tasks (Chiang et al., 2015).

A culmination of research now suggests that poorer contextual memory abilities are related to PTSD symptoms (Liberzon and Abelson, 2016). Contextual memory involves using environmental information to determine when one is safe and relies on the hippocampus (Acheson et al., 2011). Specifically, contextual memory deficits may be related to PTSD because people with PTSD experience fear in safe environments (e.g., a lion in a zoo is not threatening) and do not experience fear in dangerous environments (e.g., a lion in the wild is threatening) (Garfinkel et al., 2014). Fear learning paradigms are used to test contextual modulation of fear and safety. In these tasks, participants first learn that a cue (e.g., blue light) predicts a negative stimulus, such as a loud noise or electric shock when presented in a particular environment (e.g., office). Participants then go through an extinction protocol, in which they learn that the same cue (blue light) does not predict the negative stimulus (noise or shock) when presented in a different environment (e.g., living room). Thus, the office becomes a "dangerous" context and the living room becomes a "safe" context. Extinction recall (ER) later tests participants' ability to remember that the cue will not be paired with the negative stimulus in the "safe" environment (Milad et al., 2009). During ER, there is evidence that better performance is associated with greater hippocampal activation (Milad et al., 2009).

Studies investigating fear learning and extinction found correlations between PTSD symptom severity and patterns of brain activity during these tasks (Sripada et al., 2013). Greater hippocampal activity during fear conditioning and extinction learning was found to be positively correlated with severity of both overall PTSD symptoms and avoidance symptoms. While evidence suggests that ER is poorer in those with PTSD (Milad et al., 2009), it remains unclear how hippocampal activity during ER is associated with symptom severity across a continuum of those with PTSD and healthy individuals.

Additionally, in a few studies, stressful event exposure, separate from trauma exposure and subsequent PTSD, has also been related to ER performance. Animal studies have found that exposure to stressful events, such as maternal separation, is related to poorer ER (Cowan et al., 2013; Chocyk et al., 2014). Further, in humans, acute stress has been found to impair ER (Raio et. al., 2014). These questions have not yet been fully explored and there remains a lack of research investigating how exposure to different types of stress, even in healthy individuals, is related to ER performance.

This study aims to fill a gap in the current literature by examining hippocampal function during ER and its relationship with both stress exposure and PTSS, in people with and without PTSD. Based on previous findings of a positive relationship between ER performance and hippocampal activity (Milad et al., 2009), we expect to find a similar relationship across all participants (with and without PTSD). Additionally, we predict negative relationships between ER performance and stressful event exposure, and ER performance and PTSS severity. We also expect to find negative relationships between hippocampal activity and PTSS severity, and hippocampal activity and stressful event exposure.

\section{METHODS}

\section{Participants}

Participants included right-handed adults, with and without trauma exposure, between the ages of 18 and 45 years old. Participants were recruited from the University of Michigan Health System, advertisements around the University of Michigan campus, and the larger Ann Arbor, Michigan community. Recruitment efforts will continue for the next two years, as this project was part of a larger, ongoing study. The current study includes data from 22 participants (5 Non-trauma Exposed Controls (NEC), 8 Trauma Exposed Controls (TEC), and 9 with PTSD), with a mean age of 27 years old. Two participants were male (9.09 percent), and 20 were female (90.91 percent). Sixteen participants were 
Caucasian (72.73 percent), three were African-American (13.64 percent), two were Asian (9.09 percent), and one was Hispanic (4.55 percent). Of the 22 participants, five participants had unusable neuroimaging data due to processing inconsistencies in field map and physiological corrections. These participants were still included in analyses examining performance and clinical data.

\section{Selection Criteria}

In order for participants to be eligible for inclusion in the study, they had to be able to give informed consent and have normal or corrected-to-normal vision. TECs must have had a history of a criterion-A traumatic event but were free of any Axis-1 diagnoses including PTSD; NECs could have no history of a traumatic event or Axis-1 diagnoses. Participants with PTSD had a primary diagnosis of PTSD at the time of evaluation. Exclusion criteria for all groups included significant medical or neurological condition, active suicidal ideation, alcohol/drug abuse or dependence in the last year, a lifetime history of schizophrenia, bipolar disorder, mental retardation, or pervasive developmental disorder, and contraindication for MRI.

\section{Procedure}

All procedures were approved by the institutional review board at the University of Michigan and all participants provided written informed consent prior to enrollment.

\section{Visit Procedure}

Participants first came for an initial evaluation to determine eligibility, which included the Life Events Checklist for DSM5 (LEC-5; Weathers et al., 2013) to assess for history of criterion A trauma exposure and types of stressful event exposures, and the Clinician Administered PTSD Scale (CAPS-5; Weathers et al., 2013) to evaluate for PTSD symptoms. Additional diagnostic screeners were also used to rule out other psychiatric conditions. Eligible participants came to the lab on two consecutive days. During day one, participants completed a fear conditioning and extinction paradigm (Figure 1, day one), and during day two (24 hours later), participants completed an extinction recall and fear renewal paradigm (Figure 1, day two).

\section{Experimental Procedure}

During day one, participants completed a fear conditioning and extinction paradigm (Figure 1, day one) that lasted approximately one hour. Participants were fear conditioned with one of two contexts ("living room" and "office"), with two cues (orange and blue light) as conditioned stimuli (CS). A $500 \mathrm{~ms}$ white noise was used as the unconditioned stimulus (US). During fear conditioning, one context (office or library, counterbalanced) was shown for 2 to 7 seconds, followed by the CS for an additional 2 to 7 seconds, with each period controlled to last a total of 9 seconds. For CS+ (orange or blue light, counterbalanced), the US was delivered during 10 of 16 trials (60 percent) to coincide with CS offset. The

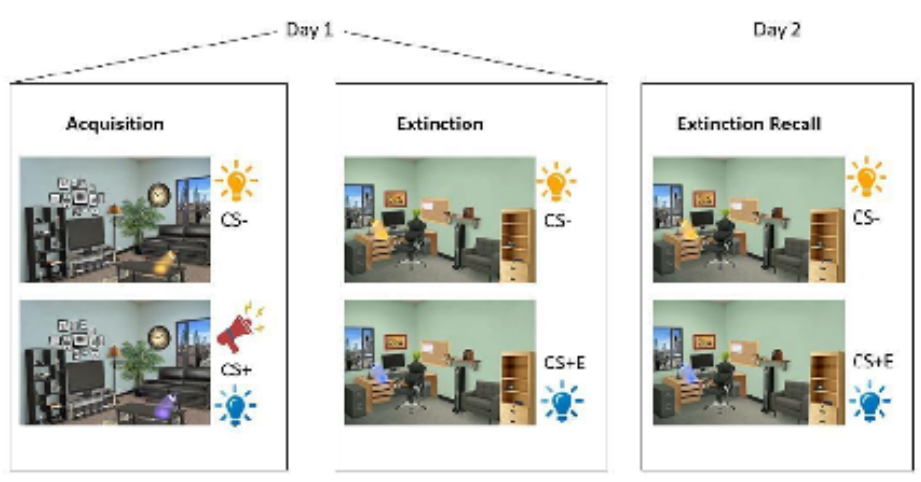

Figure 1. Fear conditioning, extinction, and extinction recall (Joshi, S. 2017). Fear conditioning contingencies (danger context and light color) were established on day one, followed by extinction in the safety context to establish CS+E. On day two, extinction recall was tested using re-presentation of the CS+ (without the presence of a loud noise) interleaved with CS- in the safety context.

CS- was presented 16 times, but never followed by the US. A fixation cross was used as the inter-trial interval, jittered for 4 to 12 seconds. Extinction followed conditioning in the other context (office or living room). Participants were shown 16 presentations of the CS+ without being followed by the US (CS+E), interleaved with 16 CS- presentations. Thus, at the end of extinction, participants should have learned that the conditioning context is "dangerous" (CS+ predicts the US in this environment), and the extinction context is "safe" (CS+E does not predict US in this environment).

On day two, participants completed the ER phase during fMRI scanning to examine hippocampal activity related to memory for prior safety learning (Figure 1, day two). During ER, the extinction context ("safe" context) from day one was presented again. Participants were shown interleaved CS+E and CS- stimuli (eight presentations each) and rated how likely they were to hear a loud noise for both the CS+ and CS-.

\section{Materials}

\section{Life Events Checklist for DSM-5}

Participants completed the Life Events Checklist for DSM-5 (LEC-5; Weathers et al., 2013) to assess how many different types of stressful life events (e.g. natural disaster, car accident, physical assault, sexual assault, etc.) participants have been exposed to throughout their lifetime. The checklist includes 17 various stressors, with the last item offering a free-form response for "other." Participants reported whether each item happened to them, was something they witnessed, learned about happening to someone they have a close relationship with, or was part of their job. Participants responded to each item with a "yes" or "no" response. Thus, the absolute number of each type of event was not assessed. This measure is limited to the assessment of whether people experienced different types of stressful events. This measure was also used to determine if a participant 
was exposed to a criterion A traumatic event for subsequent assessment for presence of PTSD. This measure was chosen because it is used along with the Clinician Administered PTSD Scale for DSM- 5 to identify the presence of stress and trauma exposure and has been shown to have good reliability and validity (LEC-5; Weathers et al., 2013).

\section{Functional Neuroimaging}

Scanning was performed using a General Electric 3T Discovery MR750 Series MRI (GE Healthcare). T1-weighted anatomical images were acquired with a 3D SPGR sequence $(F O V=256 \times 256 \mathrm{~mm}$, slice thickness $=1 \mathrm{~mm}, 0 \mathrm{~mm}$ gap). Axial slices aligned with the AC-PC plane were used for slice localization, transformation, and coregistration. Functional scans consisted of gradient echo blood oxygen level dependent (BOLD) scans (TR/TE $=2000 / 28 \mathrm{~ms}$, flip angle $=90^{\circ}, \mathrm{FOV}=192 \times 192 \mathrm{~mm}$, slice thickness $=3 \mathrm{~mm}$, $0 \mathrm{~mm}$ gap). Data was analyzed using Statistical Parametric Mapping (SPM12) for MATLAB. Images were slice-time corrected, realigned and coregistered to the structural images, normalized to the Montreal Neurological Institute (MNI) standard brain, and smoothed. We chose to use fMRI as a neuroimaging method because of its strong spatial resolution and ability to non-invasively quantify function in the brain, including the hippocampus.

\section{Expectancy Ratings}

During ER, participants were shown interleaved CS+E and CS- stimuli (eight presentations each), and rated how likely they were to hear a loud noise for both the CS+ and CS- at three points, representing an early rating one-third through, a middle rating two-thirds through, and a late rating after the final presentation. The ratings were on a scale from 1 ("not at all likely") to 5 ("definitely"). This examined whether participants could recall prior safety/extinction learning by recognizing that CS+E does not predict threat in the "safe" context. Higher expectancy ratings were indicative of worse ER performance. Consistent with other studies assessing threat and safety learning (Milad et al., 2009), we chose to use expectancy ratings as a measure of learning, as they allowed us to directly assess whether participants understood safety and threat contingencies during the tasks.

\section{Statistical Analysis}

We used SPSS to analyze participant demographics and clinical data. We ran chi-squared tests of independence to confirm that the NEC, TEC, and PTSD groups did not have statistically different distributions of race and gender, and used an ANOVA to confirm that the mean age was not statistically different between the groups. To test the hypothesis that stressful event exposure is related to PTSD symptoms across all participants, we ran a correlation in SPSS to look at the relationship between LEC-5 and CAPS scores. To understand if PTSS severity and/or stressful event exposure were related to ER, we ran correlations between CAPS/

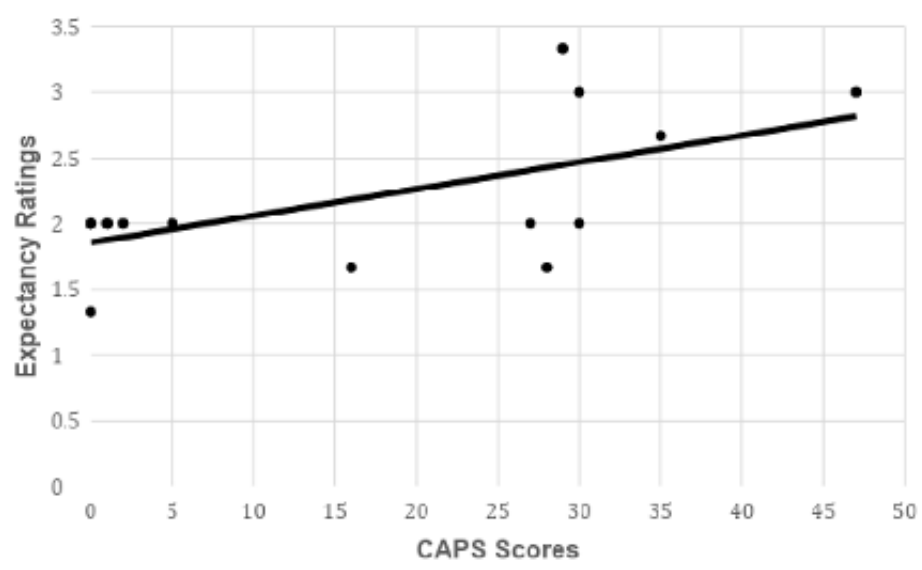

Figure 2. Positive relationship between PTSD symptom severity and expectancy ratings.

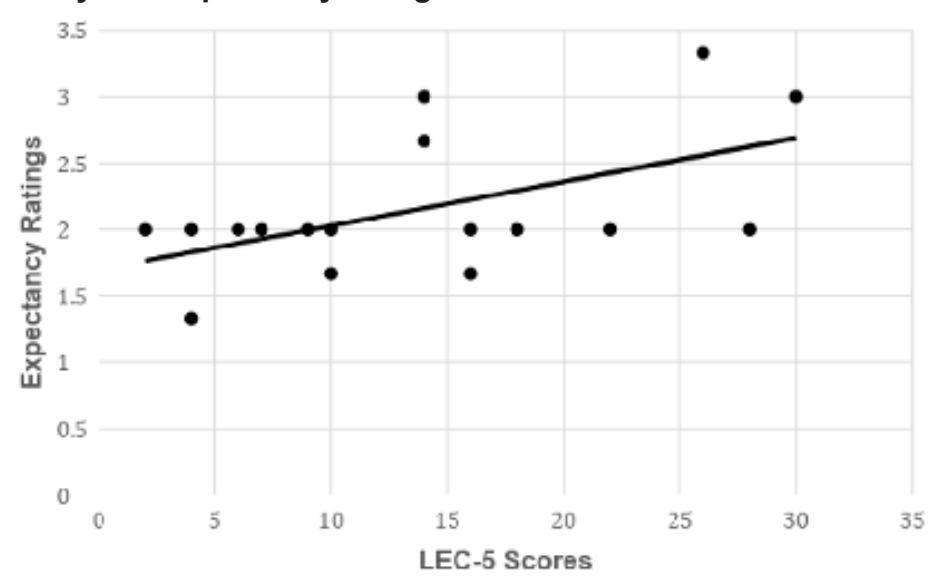

Figure 3. Positive relationship between stressful event exposure and expectancy ratings.

LEC-5 and expectancy ratings to look at the relationship between PTSS/stressful event exposure and ER performance.

\section{MRI Data Processing and Analysis}

We utilized SPM12 for MATLAB for fMRI analyses. We used an anatomical mask from the Automatic Anatomical Labeling (AAL) atlas for SPM to define the hippocampus as our region of interest (ROI) for all fMRI analyses. To create activation maps, we created a contrast to compare $C S+E$ to the implicit baseline (fixation cross). Images were thresholded at $p<0.001$, uncorrected. We used small volume family-wise error corrections (FWE SVC) within the anatomical hippocampus to correct for multiple comparisons.

To determine if hippocampal activation was related to ER performance, we extracted beta weights for each run of ER from the AAL hippocampal mask and submitted them to correlations with expectancy ratings from each run in SPSS. To test the hypothesis that PTSD symptoms are negatively related to hippocampal activity during ER, we ran a correlation between CAPS- 5 scores and activation in the hippocampal ROI. To test the hypothesis that stressful-event exposure is negatively related to hippocampal activity during the ER 
A
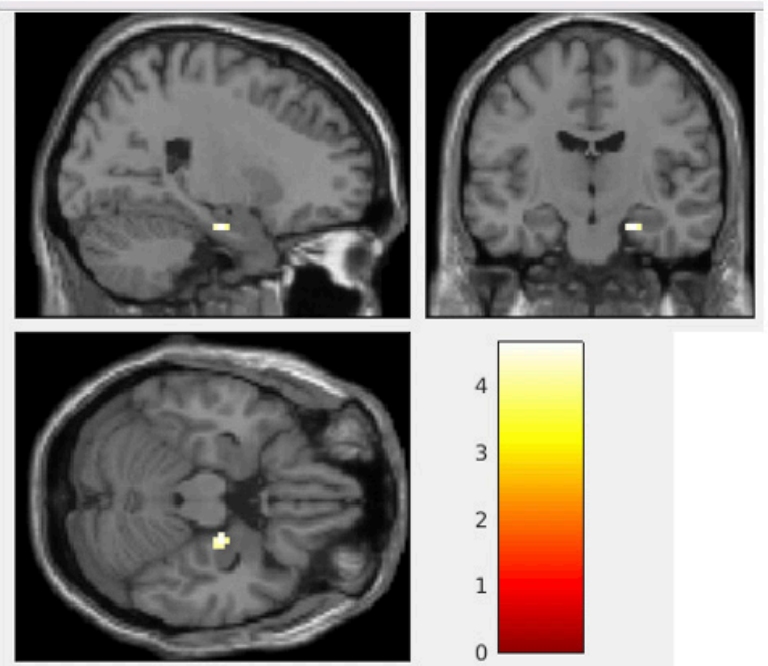

D

Figure 4. Trend towards a negative relationship between hippocampal activity during CS+E and PTSD symptom severity in all participants $(\boldsymbol{p}<0.063$ FWE SVC). 4 a. Sagittal view of the brain $4 \mathrm{~b}$. Coronal view of the brain. $4 \mathrm{c}$. Ventral view of the brain. $4 \mathrm{~d}$. Scale indicating strength of relationship.

task, we also ran a correlation between LEC-5 scores and activation in the hippocampal ROI. We chose to use correlations because our hypotheses involved examining relationships between continuous variables. Specifically, we were focused on understanding how the amount of stress/trauma exposure and PTSS severity was associated with safety memory and underlying hippocampal function across individuals with and without PTSD.

\section{RESULTS}

\section{Demographic and Clinical}

Chi-squared tests of independence showed that there were no significant differences between groups in race, $X^{2}(6)=$ 3.37 or gender distribution, $X^{2}(2)=3.85$. An ANOVA found no significant differences in age between the groups, $F(2$, 19) $=.576$.

CAPS scores across the entire sample ranged from 0 to $51(M=11.56, S D=15.25)$. An ANOVA found significant differences in CAPS scores between groups, $F(2,19)=$ $50.463, p<0.0001$. The PTSD group had significantly higher CAPS scores $(M=32.00, S D=6.76)$ than the NEC group $(M$ $=3.17, S D=6.80 ; \mathrm{t}(13)=8.074, p<0.0001)$, and the TEC group $(M=4.43, S D=5.74 ; \mathrm{t}(14)=8.62, p<0.0001)$. The TEC group did not have significantly different CAPS scores than the NEC group $(t(24)=2.01)$. LEC- 5 scores ranged from 2 to $30(M=12.78, S D=7.97)$, and an ANOVA found no significant differences in LEC-5 scores between groups, $F(2,19)=1.431$.

Hippocampal Function during Extinction Recall

We did not observe a significant relationship between hippocampal activity and expectancy ratings $(r(25)=0.016)$
A
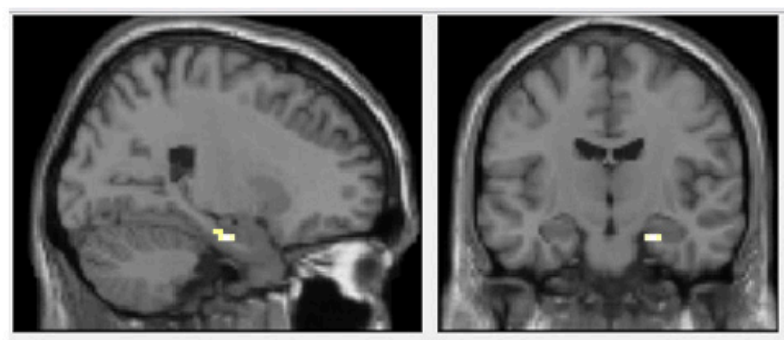

B
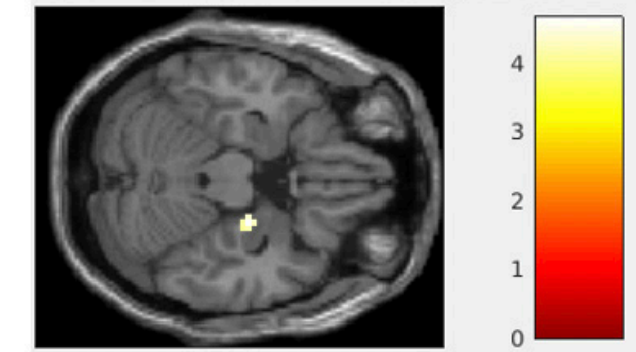

D

Figure 5. Trend towards a negative relationship between hippocampal activity during CS+E and LEC- 5 scores in all participants $(\boldsymbol{p}<0.064$ FWE SVC). 5a. Sagittal view of the brain $5 b$. Coronal view of the brain. $5 \mathrm{c}$. Ventral view of the brain. $5 \mathrm{~d}$. Scale indicating strength of relationship.

A
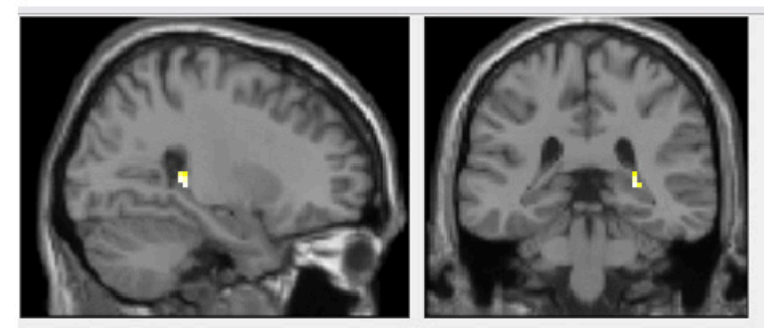

B

C
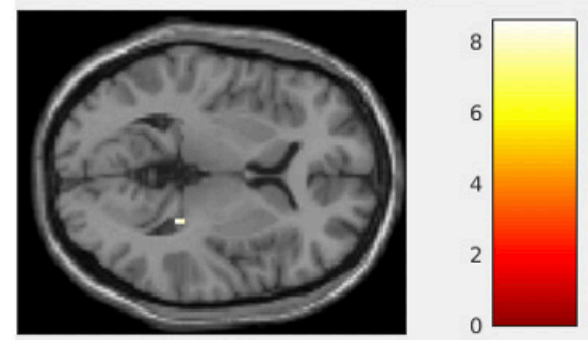

D

Figure 6. Significant negative relationship between hippocampal activity during $C S+E$ and CAPS scores in participants with PTSD ( $p<0.034$ FWE SVC). 6a. Sagittal view of the brain $6 b$. Coronal view of the brain. $6 \mathrm{c}$. Ventral view of the brain. $6 \mathrm{~d}$. Scale indicating strength of relationship.

during ER. There was a significant positive relationship between CAPS scores and expectancy ratings $(r(16)=0.677$, $p=0.008$; Figure 2), and between LEC-5 scores and expectancy ratings $(r(16)=0.656, p=0.025$; Figure 3$)$.

When participants were presented with the CS+ in the safe context $(C S+E)$, there was a trend toward significant and negative relationships between hippocampal activity and CAPS scores ( $p<0.063$ FWE SVC; $24,-13,-22$; Figure $4)$, and hippocampal activity and LEC-5 scores $(p<0.064$ FWE SVC; 24, -13, -22; Figure 5). When examining only participants with PTSD, the negative relationship between 
hippocampal activity during CS+E trials and CAPS scores was significant $(p<0.034$ FWE SVC; peak voxel 27, -7, -25; Figure 6).

\section{DISCUSSION}

The purpose of this study was to examine relationships between hippocampal activation during ER, stressful event exposure, PTSS severity, and ER performance. We predicted that ER performance would be positively related to hippocampal activation and negatively related to stress exposure and PTSS severity. Additionally, we hypothesized that there would be negative relationships between hippocampal activation during ER and (1) stressful event exposure, and (2) PTSS severity.

During ER, participants were only exposed to the conditioned stimulus in the "safe" context (Figure 1). Our results found no significant relationships between expectancy ratings and hippocampal activation during ER, which is inconsistent with previous findings (Milad et. al., 2009). It is possible that there are other factors moderating this relationship that have not been identified. Additionally, it is possible that as our sample size increases, we will see a similar relationship as described in previous research.

The relationship between CAPS-5 scores and expectancy ratings, such that greater PTSS severity was associated with higher expectancy ratings (poorer performance/ less safety memory), suggests that people with less severe PTSS demonstrate better ER. These relationships were seen across all participants, highlighting ways in which subclinical symptoms may still be related to differences in fear learning and extinction. The relationship between LEC-5 scores and ER performance was also negative, such that exposure to more types of stressful events was associated with poorer ER performance. This finding builds upon prior studies examining ER performance and stress exposure in animals (Cowan et al., 2013; Chocyk et al., 2014), and points to a similar relationship in humans. The relationship between LEC- 5 scores and task performance was also present across all participants, and not all the events participants reported through the LEC-5 were considered criterion-A traumas (American Psychiatric Association, 2013). This indicates that the relationship between safety memory and stressful event exposure is present across diagnosis and trauma-exposure, and that increased exposure to stressful events, not only traumatic events, is related to poorer safety memory.

In addition to the relationships between LEC-5, CAPS, and task performance, we also examined the relationships between LEC-5, CAPS, and hippocampal activity during ER. When considering the sample as a whole, there was an overall trend toward negative relationships between hippocampal activation and both LEC- 5 and CAPS scores, suggesting that more PTSS and stressful event exposure were both associated with less activation in the hippocampus. While these findings did not survive correction, their presence in a small sample indicates that these relationships may be significant with further data collection. Additionally, these relationships may suggest that subclinical PTSD symptoms and exposure to stressful events by healthy individuals (regardless of whether the stressful events meet criterion-A), may be connected to differences in safety memory. When examining only the group with PTSD, the negative relationship between CAPS scores and hippocampal activity was significant. This indicates that within those who have PTSD, people with more severe symptoms display less hippocampal activity when attempting to recall prior safety learning. PTSD symptoms and reactions to stressful and/or traumatic events are on a continuum (Suri and Vaidya, 2015; Luoni et. al., 2018), and our findings suggest that stress and trauma exposure is associated with differences in safety memory across the spectrum of individuals with and without PTSD.

\section{Limitations and Future Directions}

There were several limitations of this study that should be considered when interpreting our findings. The sample size was small and replication of these results with a larger sample is needed. Across all participants, the relationship between CAPS scores and hippocampal activity was trending toward significance. It is possible that further recruitment will allow for a greater variation in CAPS scores, providing us with the statistical power to detect any relationship between symptoms and hippocampal activity during ER that may be present, regardless of diagnosis or trauma exposure. Our hypotheses and neural activation analyses were limited to the hippocampus, so we cannot say if there were other regions involved in ER that may relate to stress exposure or PTSD symptoms. Investigating relationships between stress and other brain regions could help to further understand neural differences associated with trauma-exposure and/ or PTSD diagnosis. Additionally, the LEC-5 measure specifically looks at the number of types of stressful events to which someone has been exposed, not how many total lifetime stressful events someone has experienced. Using a different measure, such as the Life Stressor Checklist (LSC-R) (Wolfe et al., 1997), which assesses total number of stressful events, could help to examine the relationship between total stressful event exposure and hippocampal activation during ER in future work. Finally, future studies should use longitudinal designs to examine how the relationships between stress exposure/PTSS and hippocampal activity may look different as levels of stress exposure and the severity of PTSS change. Understanding how these relationships look longer-term in both healthy and clinical populations allows us to better comprehend the nature of these relationships and any potential translational clinical applications.

Our results demonstrate the importance of looking at PTSS on a continuum, as there are differences in hippocampal activity and fear learning performance related to stress exposure and PTSS even in healthy participants. Our find- 
ings surrounding the negative relationship between PTSS severity and hippocampal activity point to neural differences related to symptoms experienced following stressful events. This relationship implies that these relationships are present even in those who are experiencing PTSS below the diagnostic threshold. This finding may point to the need for broader access to care for those who do not meet the full criteria for PTSD but are experiencing symptoms related to stressful event exposure. Overall, our findings highlight the need for further research including individuals with and without both trauma exposure and PTSD diagnosis when considering stressful event exposure and symptoms in relation to memory and neural function. Trauma exposure and PTSD symptoms are on a continuum, rather than neatly falling into different categories (Luoni et. al., 2018; Suri and Vaidya, 2015). It is important that we recognize this and consider the relationships between all levels of these continuums, neural function, and safety memory.

\section{REFERENCES}

Acheson, D. T., Gresack, J. E. and Risbrough, V. B. (2011). 'Hippocampal dysfunction effects on context memory: possible etiology for posttraumatic stress disorder', Neuropharmacology, 62(2), 674-685, available: doi:10.1016/j.neuropharm.2011.04.029.

Admon, R., Leykin, D., Lubin, G., Engert, V., Andrews, J., Pruessner, J. and Hendler, T. (2012). 'Stress-induced reduction in hippocampal volume and connectivity with the ventromedial prefrontal cortex are related to maladaptive responses to stressful military service', Human Brain Mapping, 34(11), 2808-2816, available: doi:10.1002/hbm.22100.

American Psychiatric Association. (2013). Diagnostic and Statistical Manual of Mental Disorders (5th ed.). Arlington, VA, American Psychiatric Publishing.

Apfel, B. A., Ross, J., Hlavin, J., Meyerhoff, D. J., Metzler, T. J., Marmar, C. R., Weiner, M.W., Schuff, N. and Neylan, T. C. (2011). 'Hippocampal volume differences in gulf war veterans with current versus lifetime posttraumatic stress disorder symptoms', Biological Psychiatry, 69(6), 541-548, available: doi:10.1016/j.biopsych.2010.09.044.

Breslau, N. (2009). 'The epidemiology of trauma, PTSD, and other posttrauma disorders', Trauma, Violence, and Abuse, 10(3), 198-210, available: doi:10.1177/1524838009334448.

Carrión, V. G., Haas, B. W., Garrett, A., Song, S. and Reiss, A. L. (2009). 'Reduced hippocampal activity in youth with posttraumatic stress symptoms: an fMRI study', Journal of Pediatric Psychology, 35(5), 559-569, available: doi:10.1093/jpepsy/jsp112.

Chocyk, A., Przyborowska, A., Makuch, W., Majcher-Maślanka, I., Dudys, D. and Wędzony, K. (2014). 'The effects of early-life adversity on fear memories in adolescent rats and their persistence into adulthood', Behavioural Brain Research. 264, 161-172, available: doi:10.1016/j.bbr.2014.01.040.

Chiang, J. J., Taylor, S. E., Bower, J. E. (2015). 'Early adversity, neural development, and inflammation', Developmental Psychobiology, 57(8), 887-907, available: doi:10.1002/dev.21329

Cowan C. S., Callaghan B. L. and Richardson R. (2013). 'Acute early-life stress results in premature emergence of adult-like fear retention and extinction relapse in infant rats', Behavioral Neuroscience, 127(5), 703-711, available: doi:10.1037/a0034118.

Dickie, E. W., Brunet, A., Akerib, V. and Armony, J. L. (2008). 'An fMRI investigation of memory encoding in PTSD: influence of symptom severity', Neuropsychologia, 46(5), 1522-1531, available: doi:10.1016/j. neuropsychologia.2008.01.007.

Garfinkel, S. N., Abelson, J. L., King, A. P., Sripada, R. K., Wang, X., Gaines, L. M. and Liberzon, I. (2014). 'Impaired contextual modulation of mem- ories in PTSD: an $\mathrm{fMRI}$ and psychophysiological study of extinction retention and fear renewal', The Journal of Neuroscience, 34(40), 12425-13442, available: doi:10.1523/JNEUROSCI.4287-13.2014.

Gilbertson, M. W., Shenton, M. E., Ciszewski, A., Kasai, K., Lasko, N. B., Orr, S. P. and Pitman, R. K. (2002). 'Smaller hippocampal volume predicts pathologic vulnerability to psychological trauma', Nature Neuroscience, 5(11), 1242-1247, available: doi:10.1038/nn958.

Gurvits, T. V., Shenton, M. E., Hokama, H., Hirokazu, O., Lasko, N. B., Gilbertson, M. W., Orr, S. P., Kikinis, R., Jolesz, F. A., McCarley, R.W. and Pitman, R. K. (1996). 'Magnetic resonance imaging study of hippocampal volume in chronic, combat-related posttraumatic stress disorder', Biological Psychiatry, 40(11), 1091-1099, available: doi:10.1016/ S0006-3223(96)00229-6.

John, R. A. and Duval, E. R. (2018). 'The relationship between cumulative stress and pattern separation and pattern completion performance', The Undergraduate Journal of Psychology at Berkeley, 11, 26-35.

Joshi, S. (2017). 'Fear learning images', [Electronic image]

Liberzon, I. and Abelson, J. L. (2016). 'Context processing and the neurobiology of post-traumatic stress disorder', Neuron, 92(1), 14-30, available: doi:10.1016/j.neuron.2016.09.039.

Logue, M. W., van Rooij, S. J. H., Dennis, E. L., Davis, S. L., Hayes, J. P., Stevens, J. S., Densmore, M., Haswell, C. C., Ipser, J., Koch, S. B. J., Korgaonkar, M., Lebois, L. A. M., Peverill, M., Baker, J. T., Boedhoe, P. S. W., Frijling, J. L., Gruber, S. A., Harpaz-Rotem, I., Jahanshad, N., Koopowits, S., Levy, I., Nawijn, L., O'Connor, L., Olff, M., Salat, D. H., Sheridan, M. A., Spielberg, J. M., van Zuiden, M., Winternitz, S. R., Wolff, J. D., Wolf, E. J., Wang, X., Wrocklage, K., Abdallah, C. G., Bryant, R. A., Geuze, E., Jovanovic, T., Kaufman, M. L., King, A. P., Krystal, J. H., Lagopoulos, J., Bennett, M., Lanius, R., Liberzon, I., McGlinchey, R. E., McLaughlin, K. A., Milberg, W. P., Miller, M. W., Ressler, K. J., Veltman, D. J., Stein, D. J., Thomaes, K., Thompson, P. M. and Morey, R. A. (2017). 'Smaller hippocampal volume in posttraumatic stress disorder: a multi-site ENIGMA-PGC study: subcortical volumetry results from posttraumatic stress disorder consortia', Biological Psychiatry, 83(3), 244-253, available: doi:10.1016/j.biopsych.2017.09.006

Luoni, C., Agosti, M., Crugnola, S., Rossi, G. and Termine, C. (2018). 'Psychopathology, dissociation and somatic symptoms in adolescents who were exposed to traumatic experiences', Frontiers in Psychology, 9, 2390, available: doi:10.3389/fpsyg.2018.02390.

Milad, M. R., Pitman, R. K., Ellis, C. B., Gold, A. L., Shin, L. M., Lasko, N. B., Zeidan, M. A., Handwerger, K., Orr, S. P. and Rauch, S. L. (2009). 'Neurobiological basis of failure to recall extinction memory in posttraumatic stress disorder', Biological Psychiatry, 66(12), 1075-1082, available: doi:10.1016/j.biopsych.2009.06.026.

Qing, Z. and Gong, G. (2016). 'Size matters to function: Brain volume correlates with intrinsic brain activity across healthy individuals', Neurolmage, 139, 271-278, available: doi:10.1016/j.neuroimage.2016.06.046.

Raio, C. M., Brignoni-Perez, E., Goldman, R. and Phelps, E. A. (2014). 'Acute stress impairs the retrieval of extinction memory in humans', Neurobiology of Learning and Memory, 112, 212-221, available: doi:10.1016/j.nlm.2014.01.015.

Sripada, R. K., Garfinkel, S. N. and Liberzon, I. (2013). 'Avoidant symptoms in PTSD predict fear circuit activation during multimodal fear extinction', Frontiers in Human Neuroscience, 7, 672, available: doi:10.3389/ fnhum.2013.00672.

Suri, D. and Vaidya, V. A. (2015). 'The adaptive and maladaptive continuum of stress responses - a hippocampal perspective', Reviews in the Neurosciences, 26(4), 415-442, available: doi:10.1515/revneuro-2014-0083.

Weathers, F. W., Blake, D. D., Schnurr, P. P., Kaloupek, D. G., Marx, B. P. and Keane, T. M. (2013). The Clinician-Administered PTSD Scale for DSM-5 (CAPS-5).

Weathers, F. W., Blake, D. D., Schnurr, P. P., Kaloupek, D. G., Marx, B. P. and Keane, T. M. (2013). The Life Events Checklist for DSM-5 (LEC$5)$. 
Wolfe, J., Kimerling, R., Brown, P., Chrestman, K. and Levin, K. (1997). The Life Stressor Checklist-Revised (LSC-R).

Woon, F. L., Sood, S. and Hedges, D. W. (2010). 'Hippocampal volume deficits associated with exposure to psychological trauma and posttraumatic stress disorder in adults: A meta-analysis', Progress in Neuropsychopharmacology \& Biological Psychiatry, 34(7), 1181-1188, available: doi:10.1016/j.pnpbp.2010.06.016.

Xie, H., Erwin, M. C., Elhai, J. D., Wall, J. T., Tamburrino, M. B., Brickman, K. R., Kaminski, B., McLean, S. A., Liberzon, I. and Wang, X. (2017). 'Relationship of hippocampal volumes and posttraumatic stress disorder symptoms over early posttrauma periods', Biological Psychiatry: Cognitive Neuroscience and Neuroimaging. 3(11), 968-975, available: doi:10.1016/j.bpsc.2017.11.010. 